\title{
Rinolitíase: Revisão de Literatura a Propósito de Dois Casos Clínicos
}

\section{Rhinolithiasis: Two Clinical Cases and Review of the Literature}

Ana Campos ${ }^{1}$, José Pais ${ }^{1}$ Isaulina Barreto², Cristina Caroça ${ }^{3}$, João Paço ${ }^{4}$

\section{RESUMO}

A rinolitíase é uma patologia rara e pouco descrita na literatura. Um rinólito é uma massa calcificada das fossas nasais. Pode ser um achado acidental num doente assintomático ou ser causa de rinossinusite crónica e de destruição das fossas nasais e dos seios perinasais. É um diagnóstico a ter em conta nos casos de obstrução nasal e rinorreia unilaterais, sendo a endoscopia nasal e a tomografia computorizada fundamentais para o diagnóstico. O tratamento implica a sua remoção cirúrgica, podendo ser realizado sob anestesia local ou geral, geralmente por via endoscópica. Os autores apresentam dois casos clínicos que ilustram ambos os espectros desta condição e fazem uma revisão bibliográfica sobre o tema.

PALAVRAS-CHAVE: Litíase; Obstrução Nasal; Pólipos Nasais

1. Médico de Formação Específica de Otorrinolaringologia do Hospital. CUF Infante Santo - Núcleo Académico Clínico ORL - NOVA Medical School, Lisboa, Portugal. 2. Médica do Hospital Ayres de Menezes - São Tomé e Príncipe. 3. Médica especialista de Otorrinolaringologia do Hospital CUF Infante Santo - Núcleo Académico Clínico ORL - NOVA Medical School, Lisboa, Portugal; Professora auxiliar convidada da Nova Medical School - Faculdade de Ciências Médicas, Lisboa, Portugal. 4. Diretor do serviço de Otorrinolaringologia do Hospital CUF Infante Santo - Núcleo Académico Clínico ORL - NOVA Medical School, Lisboa, Portugal; Diretor Clínico do Hospital CUF Infante Santo - Núcleo Académico Clínico ORL - NOVA Medical School, Lisboa, Portugal. 


\section{ABSTRACT}

Rhinolithiasis is a rare condition and seldom referred in literature. Rinolith is a calcified mass in the nasal cavity. It can be an incidental finding in an asymptomatic patient or a cause of chronic rhinosinusitis with nasal cavity and paranasal sinuses destruction. It is a differential diagnosis in patients suffering from persistent rhinorrhea and nasal obstruction, most commonly unilateral. Nasal endoscopy and computed tomography are essential tools for the diagnosis. The treatment consists of surgical removal, which can be done under local or general anesthesia, by endoscopic or external approach. The authors present two clinical cases that illustrate different kinds of this pathology and make a literature review of it.

KEYWORDS: Lithiasis; Nasal Obstruction; Nasal Polyps

\section{INTRODUÇÃO}

A rinolitíase é uma condição rara, pouco descrita na literatura, frequentemente negligenciada ou mesmo desconhecida, e que tende a desaparecer nos países desenvolvidos. ${ }^{1,2}$

O termo rinólito corresponde a uma coleção calcária localizada nas fossas nasais, formada por deposição gradual de sais minerais em redor de um nidus, que pode ser endógeno ou exógeno. Exemplos de nidus endógenos são dentes, coágulos sanguíneos, ou fragmentos ósseos; exemplos de nidus exógenos são sementes, botões ou remanescentes de tamponamentos nasais. Estes últimos são mais comuns e a forma mais frequente de se instalarem é a via anterógrada, embora a via retrógrada também seja possível (por vómito ou tosse, por exemplo), bem como a sua formação in situ. , $^{2,3}$

A presença destes "materiais" desencadeia um processo inflamatório crónico, associado à deposição de sais de magnésio e de cálcio, formando massas de aspeto e consistência diversos, podendo ser cinzentos, castanhos ou esverdeados. Os rinólitos são geralmente unilaterais e localizam-se no pavimento da fossa nasal, ou entre o septo e o corneto inferior. ${ }^{3-6}$

A sintomatologia do evento desencadeante é muitas vezes ligeira, pelo que o doente vulgarmente se esquece. Após um período de latência, entre o aparecimento e o crescimento do rinólito, a sintomatologia mais intensa pode surgir, frequentemente obstrução nasal e rinorreia purulenta unilaterais. Cefaleia, epistaxis, anosmia, cacosmia, epífora, halitose ou edema do nariz ou face também estão descritos. Pela inespecificidade das queixas, os rinólitos localizados na região posterior das fossas nasais podem passar despercebidos, sobretudo se o exame objetivo não recorrer à endoscopia. ${ }^{6,7}$

À medida que aumenta de tamanho, o rinólito pode comprimir e/ou erodir estruturas vizinhas. Perfuração sep- tal, fístula oro-antral, invasão do seio maxilar, osteomielite e abcesso epidural são complicações possíveis. 5,8,9

Em conjunto com endoscopia nasal, a tomografia computorizada de seios perinasais (TC SPN) é o exame de eleição para o diagnóstico de rinolitíase. A imagem típica é de uma lesão hiperdensa com eventual área hipodensa no centro, correspondendo ao nidus. Além de precisar - local exato da lesão e ser útil na programação de uma eventual abordagem cirúrgica, este exame permite também identificar patologias concomitantes, como rinossinusite ou desvios do septo nasal, bem como presença de complicações associadas. ${ }^{6,10-12}$

O diagnóstico diferencial abrange lesões hiperdensas das fossas nasais e inclui pólipo calcificado, sequestro ósseo, tumores benignos (hemangioma, osteoma, fibroma ossificante) e malignos (osteossarcoma, condrossarcoma). ${ }^{4,10,11}$

\section{CASOS CLÍNICOS}

\section{CASO 1}

Trata-se de uma doente do sexo feminino, 56 anos, natural e residente em São Tomé e Príncipe, observada no hospital Ayres Menezes. Apresentava queixas de obstrução nasal bilateral, cacosmia e rinorreia fétida com vários anos de evolução. Negava sintomatologia associada, nomeadamente febre ou perda de peso.

Na endoscopia observou-se lesão granulomatosa, ocupando ambas as fossas nasais, não franqueável e abundante rinorreia purulenta (Fig. 1).

A doente fez curso de antibioterapia e corticoterapia local e sistémica. Na consulta de seguimento mantinha lesão com as mesmas características, pelo que se optou por pedir TC dos seios perinasais, que revelou volumosa lesão hiperdensa localizada nas fossas nasais, com destruição parcial do septo nasal e das paredes mediais dos 


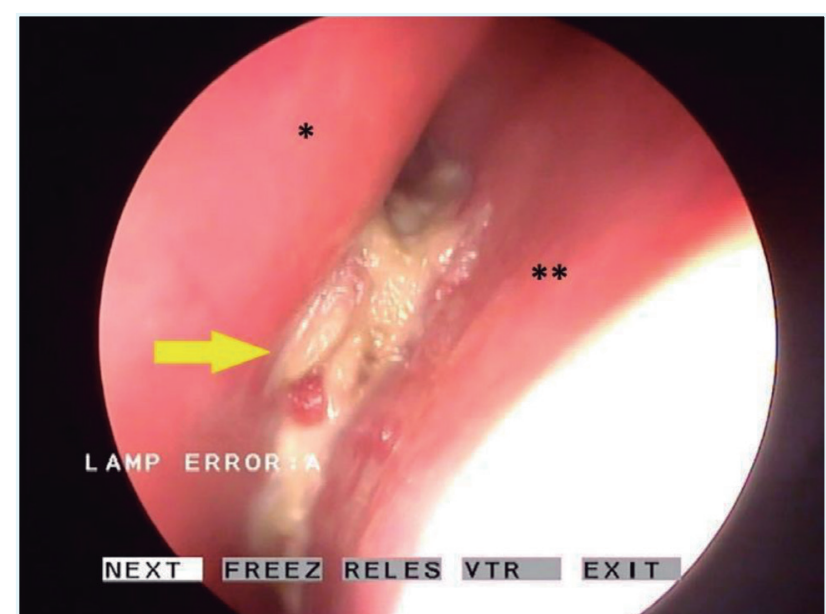

FIGURA 1. Lesão recoberta de exsudado na fossa nasal, entre o septo nasal $\left({ }^{*}\right)$ e o corneto inferior $\left({ }^{* *}\right)$

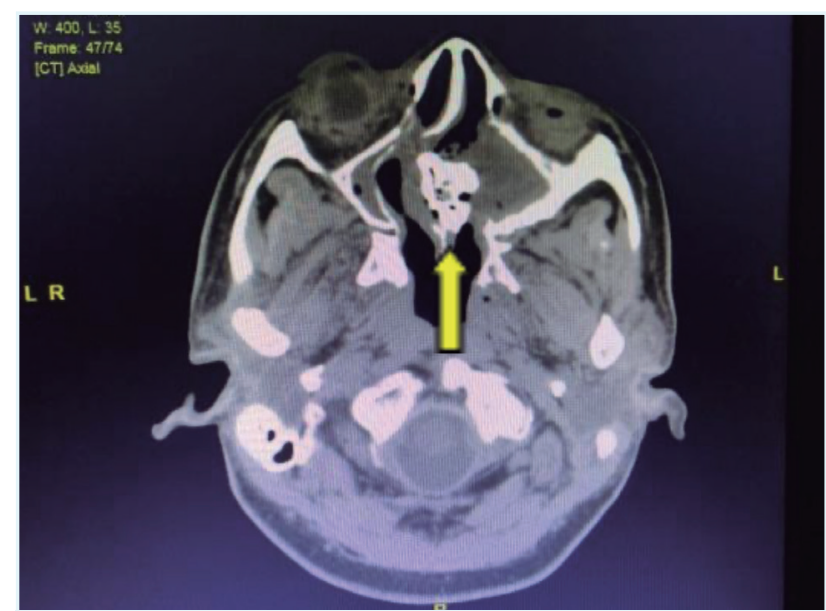

FIGURA 2. TC SPN evidenciando lesão medial de densidade óssea.

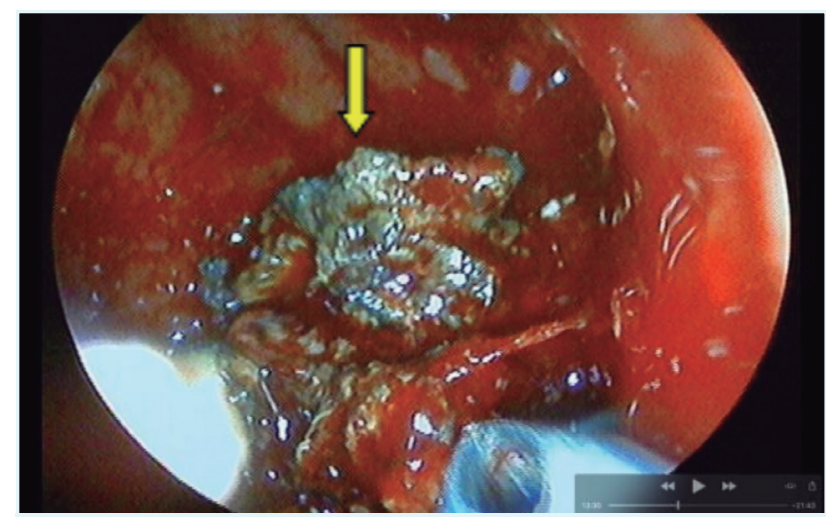

FIGURA 3. Lesão stone like da fossa nasal.

seios maxilares, e sinusite maxilar associada (Fig. 2).

Realizou-se biópsia excisional por cirurgia endoscópica nasossinusal, verificando-se massa de aspeto e consistência pétrea (Fig. 3). A lesão foi removida na sua totalidade, após fragmentação (Fig. 4). A anatomia patológica revelou massa calcificada compatível com rinólito.

No follow-up a um ano a doente apresentava-se assintomática, embora mantendo perfuração do septo nasal (Fig. 5).

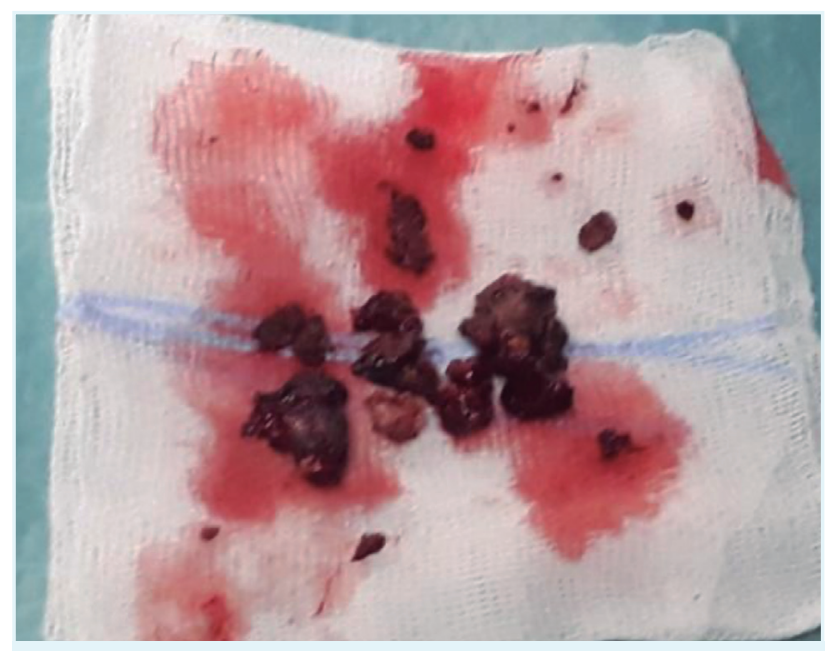

FIGURA 4. Peça cirúrgica fragmentada.

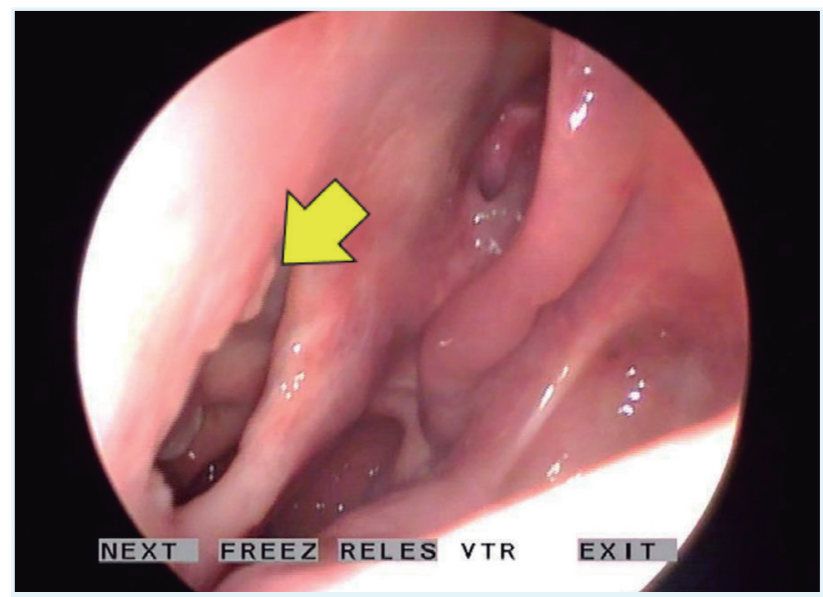

FIGURA 5. Imagem endoscópica da fossa nasal esquerda 1 ano após cirurgia, evidenciando perfuração septal.

\section{CASO 2}

Trata-se de uma doente do sexo feminino, natural do Brasil, observada no hospital CUF Infante Santo. Apresentava queixas de roncopatia e obstrução nasal direita há vários anos. Negou sintomatologia associada, nomeadamente rinorreia, febre ou perda de peso.

$\mathrm{Na}$ avaliação endoscópica observou-se, no pavimento da fossa nasal direita, massa acinzentada, de consistência pétrea, com cerca de $1,5 \mathrm{~cm}$ de maior diâmetro, sem sinais infeciosos ou inflamatórios dos tecidos circundantes (Fig. 6)

Neste caso, optou-se por remover a lesão no consultório, sob anestesia local. A anatomia patológica revelou lesão calcificada, compatível com rinólito.

\section{DISCUSSÃO}

O diagnóstico de rinolitíase é raro, mas deve ter sido em conta em doentes com queixa de obstrução nasal de longa duração, rinorreia purulenta unilateral, cefaleia crónica, halitose e/ou epistaxis recorrente. Como apre- 


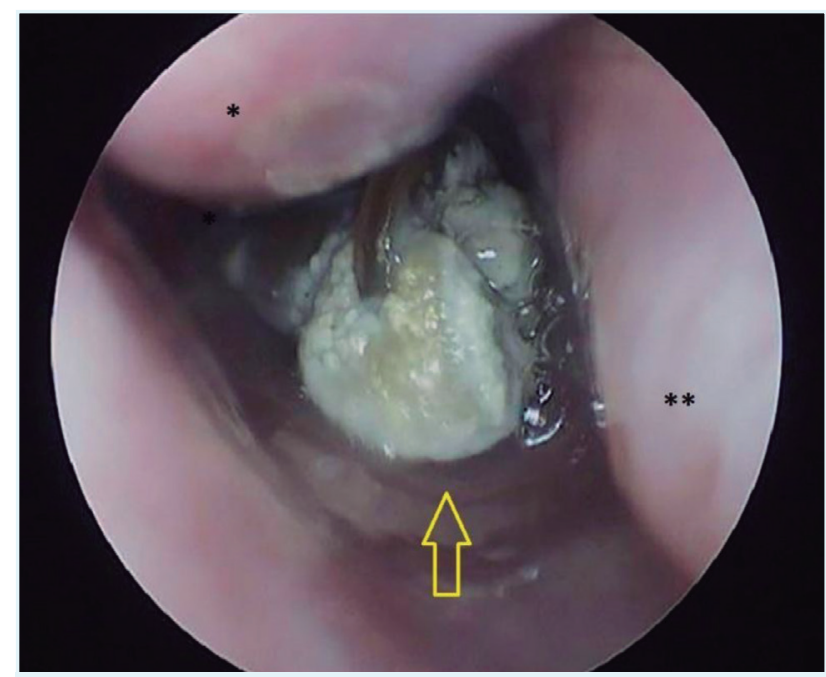

FIGURA 6. Corpo estranho na fossa nasal direita, entre o septo nasal $\left({ }^{* *}\right)$ e o corneto inferior $\left({ }^{*}\right)$.

sentado no caso 2, pode também surgir como achado acidental num doente assintomático.

A endoscopia nasal e o TC de seios perinasais são ferramentas indispensáveis para o diagnóstico, planeamento e abordagem desta patologia.

O tratamento baseia-se na excisão do rinólito e tecidos circundantes (com eventual biópsia dos mesmos), devendo a abordagem ser escolhida em função das características da patologia e do doente. Em doentes colaborantes e patologia simples poderá optar-se pela remoção sob anestesia local e com recurso à endoscopia, como no caso 2. Em doentes pouco colaborantes ou patologia avançada (existência de complicações, rinólito de grandes dimensões) poderá optar-se por cirurgia sob anestesia geral. De forma geral, a cirurgia endoscópica nasossinusal é a via preferida, mas a abordagem externa também é uma possibilidade em casos mais complexOS. ${ }^{3,6-8,11-13}$

No pós-operatório deve ser instituída antibioterapia profilática e analgesia adequada. A litotrícia, embora relatada por alguns autores, não é consensual. A recorrência é muito rara. $7,8,13,14$

\section{RESPONSABILIDADES ÉTICAS}

CONFLITOS DE INTERESSE: Os autores declaram a inexistência de conflitos de interesse na realização do presente trabalho.

FONTES DE FINANCIAMENTO: Não existiram fontes externas de financiamento para a realização deste artigo.

CONFIDENCIALIDADE DOS DADOS: Os autores declaram ter seguido os protocolos da sua instituição acerca da publicação dos dados de doentes.
CONSENTIMENTO: Consentimento do doente para publicação obtido.

PROVENIÊNCIA E REVISÃO POR PARES: Não comissionado; revisão externa por pares.

\section{ETHICAL DISCLOSURES}

CONFLICTS OF INTEREST: The authors have no conflicts of interest to declare.

FINANCING SUPPORT: This work has not received any contribution, grant or scholarship.

CONFIDENTIALITY OF DATA: The authors declare that they have followed the protocols of their work center on the publication of data from patients.

PATIENT CONSENT: Consent for publication was obtained.

PROVENANCE AND PEER REVIEW: Not commissioned; externally peer reviewed.

\section{REFERÊNCIAS}

1. Morgan J. Rhinolithiasis. J Laryngol Otol. 1957;71:331-8. doi: 10.1017/s0022215100051859

2. Lahma J, Hejjouji R, Azzam I, Oujilal A, Essakalli L. Rhinolithiasis: about an observation of a rare condition. Pan Afr Med J. 2018;31:78. doi: 10.11604/pamj.2018.31.78.16570.

3. Hadi U, Ghossaini S, Zaytoun G. Rhinolithiasis: a forgotten entity. Otolaryngol Head Neck Surg. 2002; 126:48-51. doi:10.1067/mhn.2002.121018.

4. Yildirim N, Arslanoglu A, Sahan M, Yildirim A. Rhinolithiasis: clinical, radiological, and mineralogical features. Am J Rhinol. 2008;22:78-81. doi: 10.25007air.2007.21.3112.

5. Genc S, Kahraman E, Ozel HE, Genc MG. Bilateral rhinolithiasis. J Craniofac Surg. 2011;22(86):2429. doi: 10.1097/ SCS.0b013e318231e352.

6. Ozdemir S, Akbas, Gorgulu O, Selçuk T, Sayar Ç. Rhinolithiasis: Review of 21 cases. Am J Rhinol Allergy. 2010;24:136-9. doi: 10.2500/ajra.2010.24.3553.

7. Karli R, AK M, Karli A. A different placement of the stone; rhinolithiasis. Eur Rev Med Pharmacol Sci. 2012;16:1541-5.

8. Kharoubi S. Revue générale sur les rhinolithiases. Ann Otolaryngol Chir Cervico-Faciale. 2008;125:11-7. doi: 10.1016/j. aorl.2007.12.001.

9. Dib G, Tangerina R, Abreu C, Santos R, Gregório L. Rhinolithiasis as cause of oronasal fistula. Braz J Otorhinolaryngol. 2005;71:101-3. doi: 10.1016/s1808-8694(15)31294-5.

10. Igoumenakis D, Athanasiou S, Mezitis M. A bizarre cause of extensive oronasal fistula. J Craniofc Surg. 2013;24:e:483-4. doi: 10.1097/SCS.0b013e318290336d.

11. Ozdemir S, Gorgulu O, Akbas Y, Selçuk T, Sayar H. An unusual co-presentation of rhinolithiais and squamous cell carcinoma in the nasal cavity. J Craniomaxillofac Surg. 2012;40:e137-9. doi: 10.1016/j.jcms.2011.07.008.

12. Akasakal C. Rhinolith: Examining the clinical, radiological and surgical features of 23 cases. Auris Nasus Larynx. 2019;46:542-7. doi: 10.1016/j.anl.2018. 
13. Yuca k, Caksen H, Etik O, Bayram I, Sakin YF, Dulger H, Kiris M. The importance of rigid nasal endoscopy in the diagnosis and treatment of rhinolithiais. Auris Nasus Larynx. 2006;33:1922. doi: 10.1016/j.anl.2005.05.013.

14. Mink A, Gati I, Szekely J. Nasolith removal with ultrasound lithotripsy. HNO. 1991;39:116-7. 